\title{
Predicting sensor fusion performance using theoretical models
}

\author{
B. A. Baertlein*, W.-J. Liao and D.-H. Chen \\ The Ohio State University ElectroScience Laboratory \\ 1320 Kinnear Road, Columbus, OH 43212
}

\begin{abstract}
Simple theoretical models can be constructed to study the behavior of sensor-fused systems using idealized sensor suites. Models are available for feature-level and decision-level fusion, both of which are now being used with demining sensors. These models are attractive as design tools and for estimating the expected performance of new sensor suites, since their performance can be evaluated with relatively little effort. In this paper we review some simple idealized models and their predictions for fused system performance. The data produced by demining sensors are often correlated, and the effect of correlation is explored for both feature-level and decision-level fusion.
\end{abstract}

Keywords: mine detection, sensor fusion, independence, theoretical analysis, performance prediction

\section{INTRODUCTION}

Demanding performance requirements are placed on sensors that detect hazards such as buried land mines. A high probability of detection is obviously necessary and, when the hazards are spatially disperse, as is often the case for land mines, low false alarm rates are also required. Multi-sensor data fusion is often proposed as a means of meeting these requirements, but additional resources must be allocated to develop hardware and software for a multi-sensor system, and there is no a priori guarantee that the performance of the fused suite will meet its goals.

In this work we review some basic properties of fusion that can be predicted from theoretical work. Several relatively simple idealized models can be used to estimate the performance gains achievable through fusion given individual sensor performance data such as $P_{d}$ and $P_{f a}$. We employ these theoretical results to examine the benefits achievable through fusion at the feature level and at the decision level. We compute the performance improvement (expressed as a reduction in error rate) for fusion. We also consider the important problem of correlated sensors. Many demining sensors tend to produce errors on a common set of targets, and theory allows us to predict the effect of this correlation on the performance of the fused sensor suite.

This work is organized in three major sections. In Section 2 we review some useful theoretical results for uncorrelated sensors. Expressions for the likelihood ratios of fused systems are presented for feature-level (FL) and decision-level (DL) fusion. The effects of correlation are discussed in Section 3. We present experimental data from demining sensors that suggest that correlation among demining sensors is common. The analysis shows that correlation strongly degrades performance. Concluding remarks appear in Section 4.

\section{INDEPENDENT SENSORS}

The assumption of independent sensors is relatively common in fusion studies, and it greatly simplifies the analysis. Both feature-level and decision-level fusion are addressed in what follows.

\subsection{Feature-Level Fusion}

In FL fusion target-related parameters are acquired by several sensors and merged into a feature vector. Detection is performed on the basis of the feature vector. In theory, the best features for discrimination of $K$ classes are the $K$ a posteriori probabilities. ${ }^{1}$ In practice, those probabilities are unknown, and selecting good features is frequently a problem.

Some important aspects of FL fusion can be illustrated by considering the simple case of normally distributed features. The required analysis in this case is well known. Consider the case in which $N$ features are fused into a single feature vector $\mathbf{X}$. Define hypotheses $H_{k}, k=0,1$ to be the no-mine and mine case, respectively. We assume

Corresponding author: B.A.B. (614) 292-0076 (voice), (614) 292-7297 (fax), baertlein.1@osu.edu 


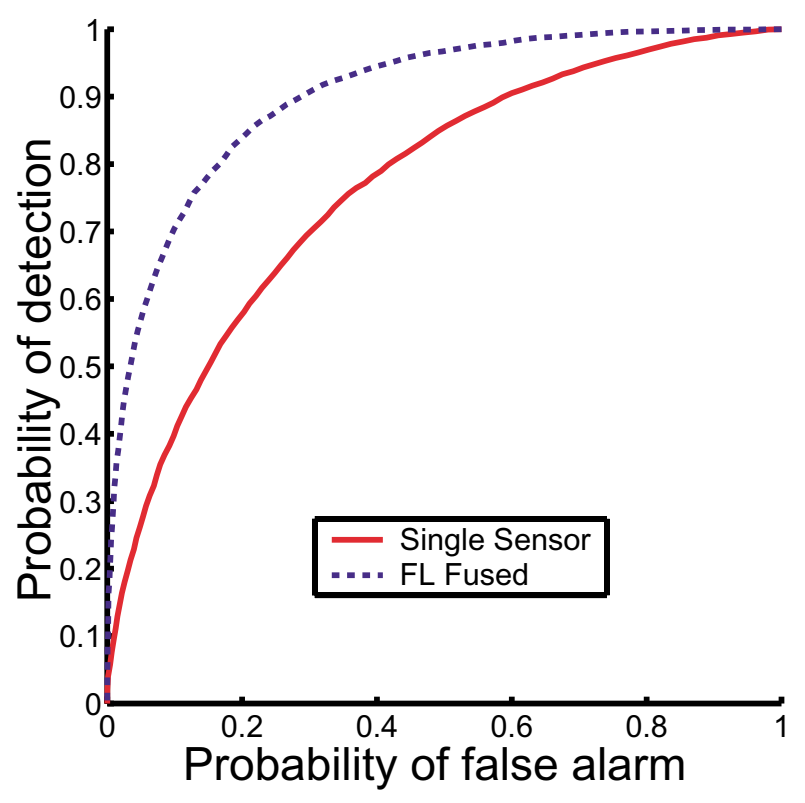

Figure 1. ROC curves for fusion of three uncorrelated identical sensors having $P_{d}=0.7$ and $P_{f a}=0.3$.

that under hypothesis $H_{k}, \mathbf{X}$ comprises independent multivariate normal deviates with mean $\mu_{k}$ and variance $\sigma_{k}^{2}$. Minimizing the Bayes cost leads to a likelihood ratio $\Lambda(\mathbf{X})=\frac{\operatorname{Pr}\left(\mathbf{X} \mid H_{1}\right)}{\operatorname{Pr}\left(\mathbf{X} \mid H_{0}\right)}$, the logarithm of which has the well-known form

$$
\ln (\Lambda(\mathbf{X}))=-\left(\mathbf{X}-\mu_{1}\right)^{T}\left(\mathbf{X}-\mu_{1}\right) /\left(2 \sigma_{1}^{2}\right)+\left(\mathbf{X}-\mu_{0}\right)^{T}\left(\mathbf{X}-\mu_{0}\right) /\left(2 \sigma_{0}^{2}\right)+N \ln \left(\sigma_{0} / \sigma_{1}\right)
$$

It is possible to express the probability density of this likelihood ratio (for both independent and correlated features to be discussed later) in a semi-analytical form, ${ }^{2}$ which yields values for $P_{d}$ and $P_{f a}$. Analogous simplifications are not possible for the DL fusion results to be presented next, and in the interest of consistency we evaluated the performance of FL fusion using Monte Carlo methods. A sequence of normal random deviates was generated for each of the hypotheses. For each random deviate we computed the likelihood ratio. For a fixed decision threshold this yields a hard decision, which can be scored, ultimately producing a ROC curve. A total of 20,000 random deviates were used to generate each of the ROC curves shown in this work.

In this work we use as a performance metric the probability of error $P_{E}$ (or the error rate) given by

$$
P_{E}=\operatorname{Pr}\left(H_{0}\right) P_{f a}+\operatorname{Pr}\left(H_{1}\right)\left(1-P_{d}\right)
$$

where $\operatorname{Pr}\left(H_{k}\right)$ are the prior probabilities, $P_{f a}$ is the false alarm probability, and $P_{d}$ is the detection probability. Legitimate objections can be raised to the use of this metric for mine detection, since it implicitly assigns equal costs to a missed detection and a false alarm. It is, however, a common standard, and the extension to other metrics is straightforward. The value of $P_{E}$ is also dependent on the decision threshold. Throughout this work we assume that any system would be operated at its minimum error point, and we use the minimum value of $P_{E}$ in our analyses. In addition, we consider only the case $\operatorname{Pr}\left(H_{0}\right)=\operatorname{Pr}\left(H_{1}\right)=0.5$.

In general, fusion produces a performance gain, which we quantify as follows:

$$
\text { Fusion gain }=\left(\text { minimum } P_{E} \text { for best single sensor }\right)-\left(\text { minimum } P_{E} \text { for fused sensor suite }\right)
$$

As an example, in Figure 1 we have plotted the performance of a FL system using three uncorrelated features each of which has a ROC curve that passes through the point $P_{d}=0.7, P_{f a}=0.3$. For this uncorrelated-feature case, fusion reduces $P_{E}$ by $11.4 \%$.

The performance improvement attainable with fusion depends on the performance of the individual sensors. This dependence is illustrated in Figure 2, where we again show the result of fusing three sensors. Two of the sensors have 
a specified error rate, while the error rate of the third sensor is varied. To reduce the number of variables involved we have imposed the added constraint $P_{d}+P_{f a}=1$ for the third sensor. As the error rate of the third sensor increases, the performance of the fused suite tends to that of a two-sensor fused system, and the third sensor provides little benefit. Conversely, as the error rate for the third sensor decreases, it tends to dominate the performance of the suite. In such cases, the contribution of the two more poorly performing sensors is reduced. This tendency is clear from Figure 2(b) where the performance gain achievable under fusion is shown. It is evident that the maximum gain is achieved when the sensors have equal error rates, although the best performance is obtained when one sensor dominates the others.

\subsection{Decision-Level Fusion}

In the simplest form of DL fusion, a vector of sensor decisions is produced by applying individual thresholds to the test statistics output by each sensor. In the interest of brevity we restrict our attention to binary decisions. The decision vector is processed to form a new fused test statistic for the sensor suite, and a (possibly) different threshold is applied to the fused test statistic. The relation between thresholds for different sensors and for the fused suite is not readily addressed theoretically, but some work in this area has appeared previously. ${ }^{3}$

Consider the case of $N$ independent sensors each of which produces a random, binary decision $u_{n}, n=1, \ldots, N$. Writing the decisions as the vector $\mathbf{U}$, the likelihood ratio for this formulation is given by

$$
\Lambda_{I}(\mathbf{U})=\frac{\operatorname{Pr}\left(\mathbf{U} \mid H_{1}\right)}{\operatorname{Pr}\left(\mathbf{U} \mid H_{0}\right)}=\prod_{n=1}^{N} \frac{\operatorname{Pr}\left(u_{n} \mid H_{1}\right)}{\operatorname{Pr}\left(u_{n} \mid H_{0}\right)}
$$

An optimum decision rule for this case was first described by Chair and Varshney ${ }^{4}$ and is developed by decomposing the above product as follows:

$$
\Lambda_{I}(\mathbf{U})=\prod_{S_{1}} \frac{\operatorname{Pr}\left(u_{n}=1 \mid H_{1}\right)}{\operatorname{Pr}\left(u_{n}=1 \mid H_{0}\right)} \prod_{S_{0}} \frac{\operatorname{Pr}\left(u_{n}=0 \mid H_{1}\right)}{\operatorname{Pr}\left(u_{n}=0 \mid H_{0}\right)}=\prod_{S_{1}} \frac{P_{d, n}}{P_{f a, n}} \prod_{S_{0}} \frac{1-P_{d, n}}{1-P_{f a, n}}
$$

where $S_{1}\left(S_{0}\right)$ denotes the set of sensors that produce $u_{n}=1\left(u_{n}=0\right)$ for a given observation of the target, and $P_{d, n}$ and $P_{f a, n}$ are the respective detection and false alarm probabilities for sensor $n$.

Taking the logarithm of this result permits it to be expressed as

$$
\ln \left(\Lambda_{I}(\mathbf{U})\right)=\sum_{n=1}^{N}\left[u_{n} w_{n}^{(1)}+\left(1-u_{n}\right) w_{n}^{(0)}\right]
$$

where

$$
\begin{aligned}
& w_{n}^{(1)}=\ln \left(\frac{P_{d, n}}{P_{f a, n}}\right) \\
& w_{n}^{(0)}=\ln \left(\frac{1-P_{d, n}}{1-P_{f a, n}}\right)
\end{aligned}
$$

are weights are defined by sensor performance data. The foregoing summation comprises a weighted voting fusion, in which the fused decision is determined from the detections and the reliability of the sensors. Note that since only $2 N$ distinct weights are involved, a finite number of values can be assumed by $\ln (\Lambda(\mathbf{U}))$. Some special cases are of interest. If the sensors have identical performance and if $P_{d, n}=1-P_{f a, n}$ then the weights satisfy

$$
w_{n}^{(1)}=-w_{n}^{(0)} \equiv w
$$

for every $n$. In this case we have

$$
\ln \left(\Lambda_{I}(\mathbf{U})\right)=-w\left[N+\sum_{n=1}^{N} u_{n}\right]
$$

If we apply the decision thresholds $0,-w N$ and $-w N / 2$ to $\ln \left(\Lambda_{I}(\mathbf{U})\right)$, this expression leads to AND, OR, and majority voting fusion, respectively. 


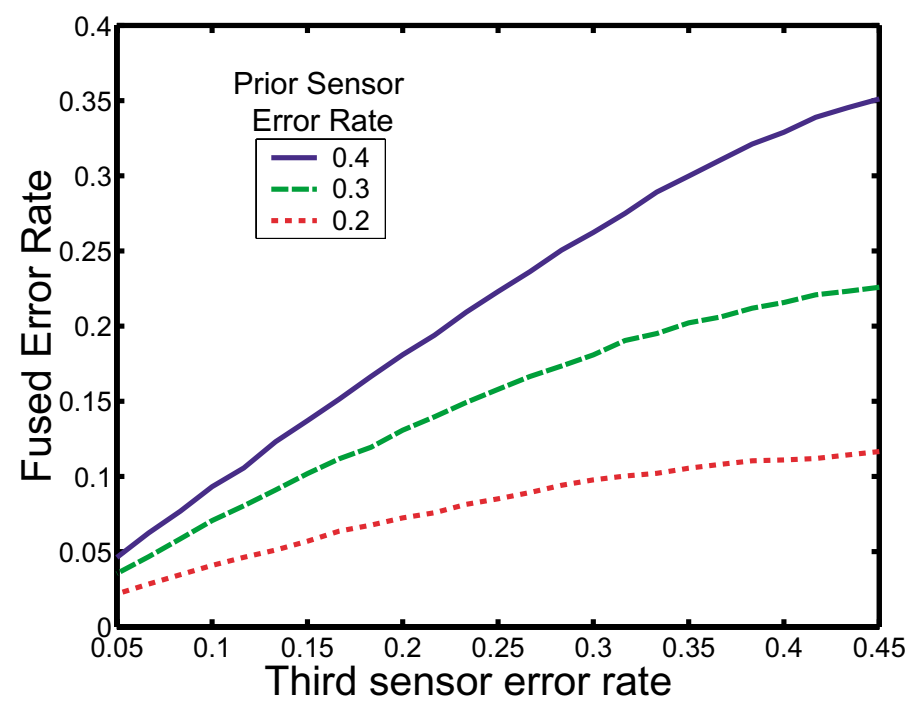

(a) Achievable performance level.

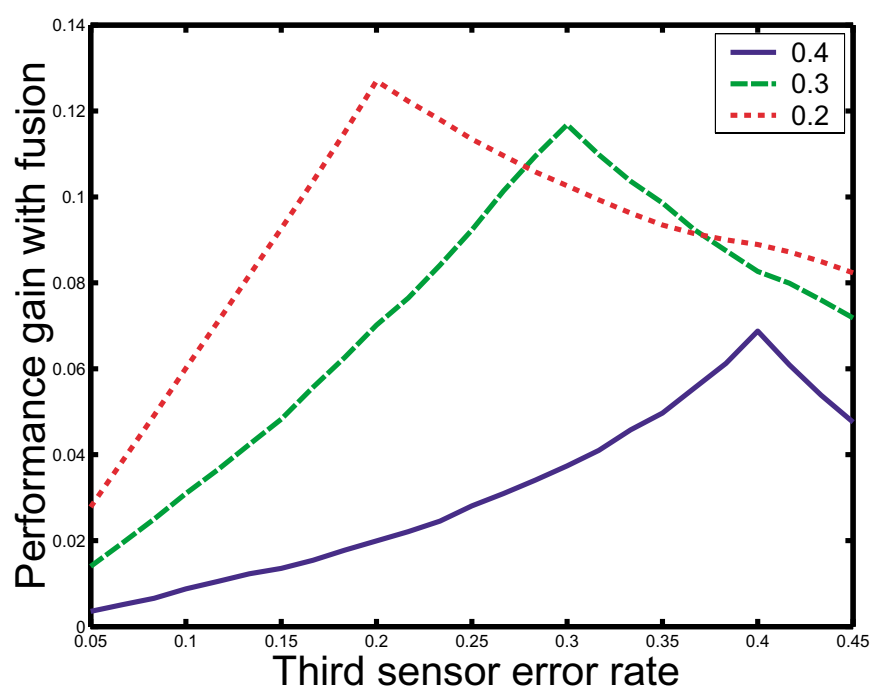

(b) Fusion-related performance gain.

Figure 2. The performance of two sensors having a specified error rate fused with a third sensor. 


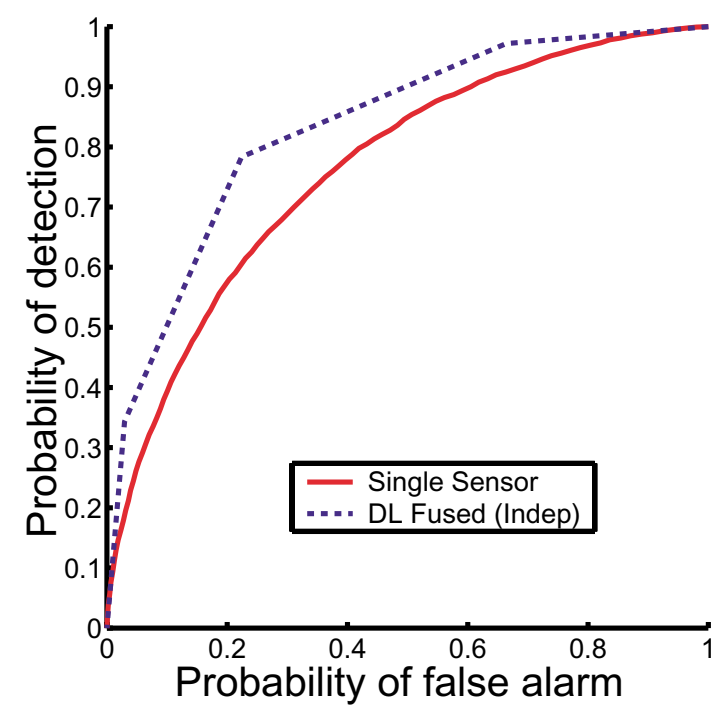

Figure 3. Performance of decision-level fusion for three sensors having $P_{d}=0.7$ and $P_{f a}=0.3$.

Simulation is again used to investigate the performance of DL fusion. Fixed thresholds can be applied to independent normal deviates yielding a sequence of independent binary random variables with specified $P_{d}$ and $P_{f a}$ values. These binary variables can be used in DL fusion performance assessments. A ROC curve for three independent sensors having $P_{d}=0.7$ and $P_{f a}=0.3$ is shown in Figure 3. For three identical sensors with the given performance values, $\ln \left(\Lambda_{I}\right)$ can take on only four discrete values corresponding to detection by none, one, two, or all sensors. The maximum gain for fusion in this case is $7.9 \%$, less than that noted for feature-level fusion above.

The gain of DL fusion is also dependent on the performance of the individual sensors. As done previously for FL fusion, we compute the performance of DL fusion algorithms for uncorrelated sensors as a function of $P_{E}$. The results are shown in Figure 4. We find that when the error rate of the third sensor falls below a specific value, there is no benefit to fusion since the best performance of the fused suite simply tracks that of the best sensor. (Recall that the decision thresholds are being selected to produce the best performance.) This finding is confirmed by the plot of fusion-related performance gain in Figure 4(b). As in the case of FLfusion, the largest gain is obtained when all three sensors have comparable error rates.

\section{CORRELATED SENSORS}

Data produced by demining sensors are frequently not independent. The outputs of these sensors are typically dominated by clutter, rather than internal noise, and a variety of clutter phenomena can produce detectable signals in multiple sensors. For example, a sufficiently large metal object will be detected by both an EMI sensor and a GPR. Similarly, a small depression in the soil will produce artifacts for IR, EMI and GPR sensors. In addition, small, non-metallic mines, are poorly detected by many sensors. In this section we use correlation as a metric for the lack of independence. In general, correlation is an imperfect measure of sensor dependence, but it is sufficient for the Gaussian features to be considered here.

\subsection{Evidence for Sensor Correlation}

A number of sensors have been used to acquire data at the JUXOCO Calibration Grid, Site 71A, Fort A. P. Hill, VA. This test site contains a grid of mines and clutter objects, the locations and identities of which are known. The site offers 125 sampling locations, of which 29 contain buried mines (both AP and AT) and 33 contain buried clutter artifacts (various metal, plastic, wood objects). The remaining sample locations are blanks (homogeneous soil with no emplaced clutter). The mines are diverse, with one to five examples of each mine. For that site we will consider data from three sensors: the GEM3 EMI sensor, a laser Doppler vibrometer, and a LWIR camera. These sensors and the processing used with them are described elsewhere, ${ }^{3,5}$ and only a brief summary is presented here. 


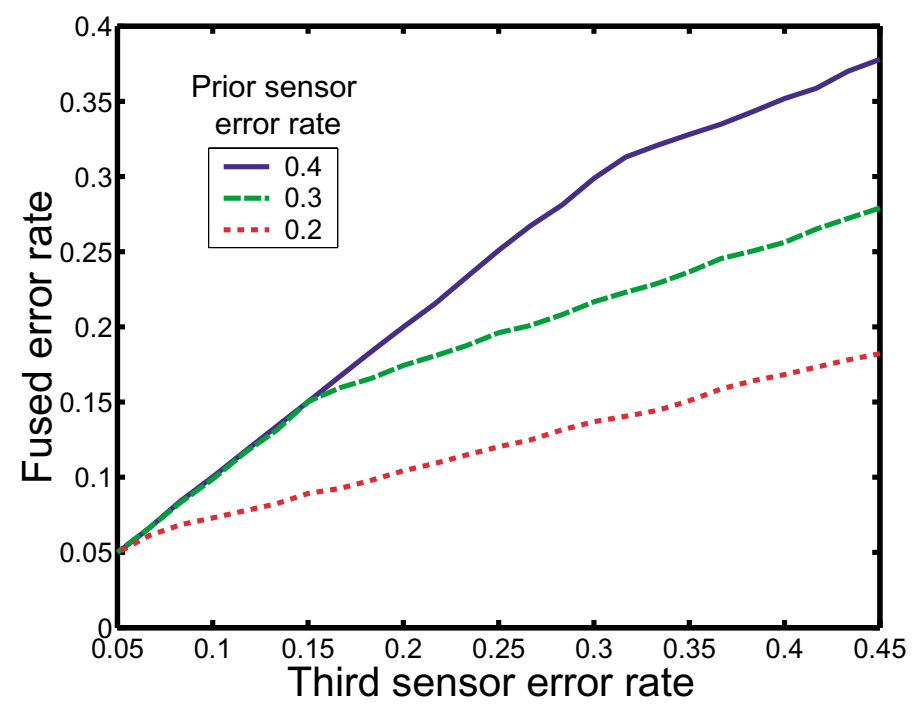

(a) Best achievable performance.

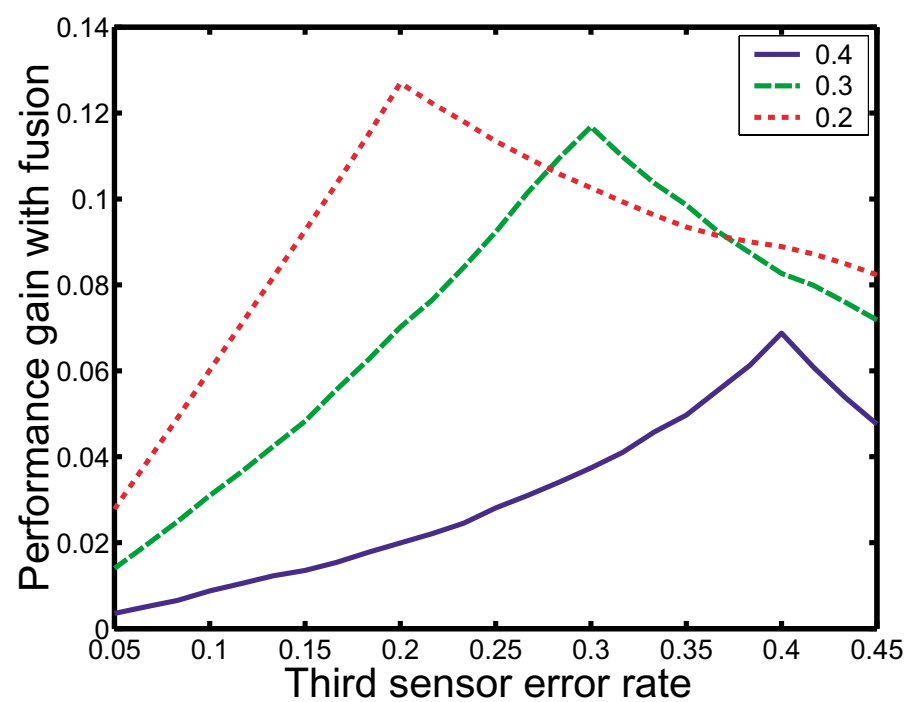

(b) Fusion-related performance gain.

Figure 4. The performance of two sensors having a specified error rate fused (at the decision level) with a third sensor. 
The GEM3 EMI data were acquired in 1999 by Duke University. The results of that collection have been documented elsewhere. ${ }^{6,7}$ In brief, those data comprise complex (I \& Q), frequency-domain measurements using the GEM3 at ten points over each mine. The cells sampled in this manner were limited to those having known mines or emplaced clutter, i.e., no blank cells were sampled. Data from these scans were used to train a classifier, which detects mines on the basis of the spatial distribution of energy. ${ }^{3}$

During 1998 The Ohio State University acquired LWIR data at the JUXOCO calibration grid. The sensor was positioned on a tripod of a known height and at a known distance from the grid cells. Data were acquired at all 125 grid cells. The sensors and processing algorithms were described in a previous work. ${ }^{3}$

The University of Mississippi has demonstrated that buried mines can be detected by vibrating the soil with acoustic speakers and sensing the vibration with a laser Doppler vibrometer (LDV). ${ }^{8,9}$ The resulting data comprise a multi-spectral image of the surface at a few tens of frequency bands and at a few tens of spatial positions. Preprocessing of the data to remove trends in the image and a CFAR algorithm used for detection have been described by Baertlein et al. ${ }^{5}$

Fusion of the EMI, LWIR, and LDV data from the JUXOCO site allow us to illustrate the concepts discussed in Sections 2.1 and 2.2. The size of the data set is small, which limits the confidence that can be placed in the results. In addition, other limitations in the data set preclude us from considering some sensor combinations: although each sensor sampled a large fraction of the 125 grid cells, each sensor also sampled a different set of locations and the intersection of these sets (i.e., the set of locations sampled by multiple sensors) is often small. In particular, only 13 of the 125 grid cells were sampled by both the GEM3 and LDV sensors. This makes it impossible to consider fusion of any sensor suite that includes both sensors. The situation is slightly better if we separately consider fusion of the LWIR data with the GEM3 and LDV data. The data available for these pairs of sensors comprises roughly 40 samples each, of which about half are mines.

In addition to the data from Fort A. P. Hill, data have been acquired by a forward-looking GPR and the LDV over a common set of targets at Yuma Proving Grounds, AZ. That data set produced a large common set of mine samples, but a smaller number of clutter samples. The details of that collection and its processing are described elsewhere. ${ }^{5}$

We define the feature-level correlation $\rho_{i j}$ between features $x_{i}$ and $x_{j}$ in the usual manner

$$
\rho_{i j}=\frac{\frac{1}{M} \sum_{m=1}^{M}\left(x_{i, m}-\mu_{i}\right)\left(x_{j, m}-\mu_{j}\right)}{\sqrt{\operatorname{var}\left(x_{i}\right) \operatorname{var}\left(x_{j}\right)}}
$$

where $\mu_{i}\left(\mu_{j}\right)$ is the mean of feature $i(j)$, and $M$ is the number of samples. A similar definition is used for the decision correlations. When a sensor produces multiple features, a classifier was used to reduce the features from each sensor to a single value.

Correlation coefficients among the sensors can be computed and are given in Tables 1 and 2 for features and decisions. We see that both features and decisions have significant correlation for some sensor combinations.

\begin{tabular}{|l|c|c|}
\hline & \multicolumn{2}{|c|}{ Correlation $\rho$} \\
\hline Sensors & $H_{1}$ & $H_{0}$ \\
\hline LWIR-GEM3 & -0.32 & 0.18 \\
\hline LWIR-LDV & 0.55 & -0.19 \\
\hline GPR-LDV & -0.23 & -0.20 \\
\hline
\end{tabular}

Table 1. Feature correlation coefficients.

\subsection{Fusion of Correlated Features}

For the case of correlated features, the log-likelihood ratio has the well-known form

$$
\ln (\Lambda(\mathbf{X}))=-\left(\mathbf{X}-\mu_{1}\right)^{T} \boldsymbol{\Sigma}_{1}^{-1}\left(\mathbf{X}-\mu_{1}\right) / 2+\left(\mathbf{X}-\mu_{0}\right)^{T} \boldsymbol{\Sigma}_{0}^{-1}\left(\mathbf{X}-\mu_{0}\right) / 2+\ln \left(\left|\boldsymbol{\Sigma}_{0}\right| /\left|\boldsymbol{\Sigma}_{1}\right|\right) / 2
$$




\begin{tabular}{|l|c|c|}
\hline & \multicolumn{2}{|c|}{ Correlation $\rho$} \\
\hline Sensors & $H_{1}$ & $H_{0}$ \\
\hline LWIR-GEM3 & -0.12 & 0.18 \\
\hline LWIR-LDV & 0.26 & -0.17 \\
\hline GPR-LDV & -0.09 & -0.07 \\
\hline
\end{tabular}

Table 2. Decision correlation coefficients.

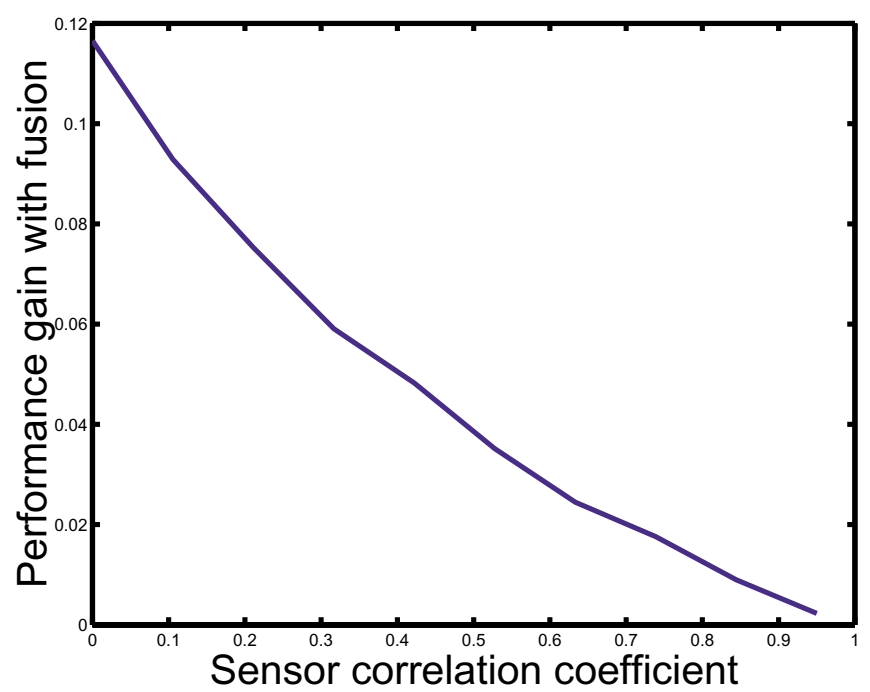

Figure 5. Performance gain of feature-level fusion for three sensors having $P_{d}=0.7$ and $P_{f a}=0.3$ and a common variable correlation level among all sensors.

where $\boldsymbol{\Sigma}_{k}$ is the covariance matrix under hypothesis $H_{k}$. Monte Carlo simulation of this case requires that we generate random deviates with the prescribed distributions, which can be done from independent, identically distributed, zeromean, unit-variance, random normal deviates. We denote the feature for sensor $n$ and trial $i$ by $x_{n}^{(i)}$. If the covariance matrix $\boldsymbol{\Sigma}$ is factored using a Cholesky decomposition,

$$
\boldsymbol{\Sigma}^{-1}=\mathbf{A}^{T} \mathbf{A}
$$

then multivariate normal deviates $y_{n}^{(i)}$ with mean $\mu$ and covariance matrix $\boldsymbol{\Sigma}$ are generated by the linear transformation

$$
\mathbf{y}^{(i)}=\mathbf{A}^{T} \mathbf{x}^{(i)}+\mu
$$

Simulations show that sensor-to-sensor correlation rapidly eliminates the benefits of fusion. This effect is illustrated in Figure 5, where we show the result of fusing three sensors mutually correlated at a given level. A theoretical interpretation of this result is that forming the matrix product with $\boldsymbol{\Sigma}_{k}^{-1}$ projects out of the data the influence of the correlated sensors, leaving only the information from the uncorrelated residual data. Comparing Figure 5 with the feature correlations shown in Table 1 suggests that FL fusion of these sensors may achieve only $30 \%$ to $65 \%$ of the performance of independent sensors.

\subsection{Fusion of Correlated Local Decisions}

In this section we consider the problem of hard decision fusion using correlated sensors. The problem has received attention previously. Aalo and Viswanathan ${ }^{10}$ performed numerical simulations of the problem and characterized how performance degrades with decreasing correlation for several different fusion strategies. Drakopoulos and Lee ${ }^{11}$ derived an optimum fusion rule for the Neyman-Pearson criterion, and used simulation to study its performance for 
a specific type of correlation matrix. Kam et al. ${ }^{12}$ considered the case in which the class-conditioned sensor-to-sensor correlation coefficients are known, and expressed the result in a compact form. Blum and Kassam ${ }^{13}$ studied the problem of locally most powerful detection for correlated local decisions.

The formulation of Kam et al. is attractive, since it permits the effects of correlation to be addressed explicitly and simply. Their formulation begins by reducing the decisions to a standard zero-mean, unit-variance form using the transformation

$$
y_{n}=\frac{u_{n}-p_{n}}{\sqrt{p_{n}\left(1-p_{n}\right)}}
$$

in which

$$
p_{n}=\operatorname{Pr}\left(u_{n}=1\right)=P_{1} P_{d, n}+P_{0} P_{f a, n}
$$

One next uses the Bahadur-Lazarsfeld (BL) polynomials $\psi_{i}(\mathbf{U})$ described by Duda and Hart. ${ }^{14}$ These polynomials comprise all possible products of the $y_{n}$ taken none, one, two, and on to $N$ at a time. Explicitly

$$
\psi_{i}(\mathbf{U})= \begin{cases}1 & i=0 \\ y_{i} & i=1 \\ y_{2} & i=2 \\ \vdots & \vdots \\ y_{N} & i=N \\ y_{1} y_{2} & i=N+1 \\ y_{1} y_{3} & i=N+2 \\ \vdots & \vdots \\ y_{N-1} y_{N} & i=N+N(N-1) / 2 \\ y_{1} y_{2} y_{3} & i=1+N+N(N-1) / 2 \\ \vdots & \vdots \\ y_{1} y_{2} \ldots y_{N} & i=2^{N}-1\end{cases}
$$

The BL polynomials can be used to expand the joint density $\operatorname{Pr}(\mathbf{U})$. If we define the density of independent variables as

$$
P_{1}(\mathbf{U})=\prod_{n=1}^{N} p_{n}^{u_{n}}\left(1-p_{n}\right)^{1-u_{n}}
$$

then using $P_{1}$ as a weighting function, the $\psi_{i}$ are orthonormal, viz:

$$
\sum_{\mathbf{U}} \psi_{i}(\mathbf{U}) \psi_{j}(\mathbf{U}) P_{1}(\mathbf{U})=\delta_{i j}
$$

where $\mathbf{V}$ ranges over all possible values of $\mathbf{U}$ and $\delta_{i j}$ is the Kronecker delta function. Using these polynomials, any probability $\operatorname{Pr}(\mathbf{U})$ can be expanded in the form

$$
\operatorname{Pr}(\mathbf{U})=P_{1}(\mathbf{U}) \sum_{i=1}^{2^{N}-1} a_{i} \psi_{i}(\mathbf{U})
$$

where the expansion coefficients are given by

$$
a_{i}=\sum_{\mathbf{V}} \psi_{i}(\mathbf{V}) \operatorname{Pr}(\mathbf{V})
$$

Equation (20) shows that the density of correlated variables $\mathbf{U}$ can be represented by the density of the independent variables $P_{1}(\mathbf{U})$ times a correction factor, which is written as a sum of BL polynomials. It was noted by Kam et al. ${ }^{12}$ that for an expansion using the normalized variables $y_{n}$, we have $a_{0}=1, a_{i}=0$ for $i=1,2, \ldots, N$ (since the $y_{i}$ are zero mean), and that for $i>N$ the $a_{i}$ are correlation coefficients, which have the form

$$
\rho_{i j k \ldots n}=\sum_{\mathbf{V}} y_{i} y_{j} \ldots y_{n} \operatorname{Pr}(\mathbf{V})
$$




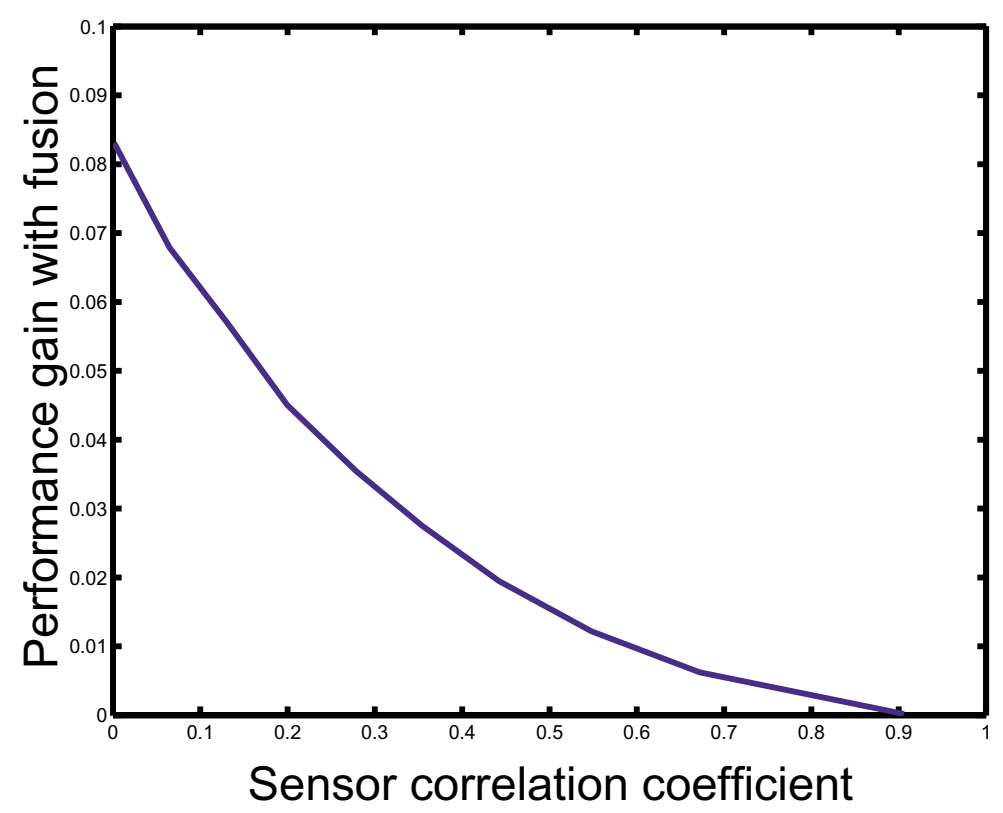

Figure 6. Performance gain for all sensors correlated at a common level.

Using these facts we can write

$$
\operatorname{Pr}(\mathbf{U})=P_{1}(\mathbf{U})\left[1+\sum_{i<j} \rho_{i j} y_{i} y_{j}+\sum_{i<j<k} \rho_{i j k} y_{i} y_{j} y_{k}+\cdots \rho_{123 \ldots N} y_{1} y_{2} \ldots y_{N}\right]
$$

This procedure can be used to formulate a likelihood ratio for correlated sensors. If we define normalized random variables $y_{n}^{(k)}$ conditioned on hypothesis $H_{k}$

$$
y_{n}^{(k)}=\frac{u_{n}-\operatorname{Pr}\left(u_{n}=1 \mid H_{k}\right)}{\sqrt{\operatorname{Pr}\left(u_{n}=1 \mid H_{k}\right)\left[1-\operatorname{Pr}\left(u_{n}=1 \mid H_{k}\right)\right]}}
$$

then the densities $\operatorname{Pr}\left(\mathbf{U} \mid H_{k}\right)$ can be expanded as above to yield the following expression for the likelihood ratio $\Lambda_{C}(\mathbf{U})$ of correlated sensors

$$
\Lambda_{C}(\mathbf{U})=\Lambda_{I}(\mathbf{U})\left[\frac{1+\sum_{i<j} \rho_{i j}^{(1)} y_{i}^{(1)} y_{j}^{(1)}+\sum_{i<j<k} \rho_{i j k}^{(1)} y_{i}^{(1)} y_{j}^{(1)} y_{k}^{(1)}+\cdots \rho_{123 \ldots N}^{(1)} y_{1}^{(1)} y_{2}^{(1)} \ldots y_{N}^{(1)}}{1+\sum_{i<j} \rho_{i j}^{(0)} y_{i}^{(0)} y_{j}^{(0)}+\sum_{i<j<k} \rho_{i j k}^{(0)} y_{i}^{(0)} y_{j}^{(0)} y_{k}^{(0)}+\cdots \rho_{123 \ldots N}^{(0)} y_{1}^{(0)} y_{2}^{(0)} \ldots y_{N}^{(0)}}\right]
$$

in which

$$
\rho_{i j \ldots n}^{(k)}=\sum_{\mathbf{V}} y_{i}^{(k)} y_{j}^{(k)} \ldots y_{n}^{(k)} \operatorname{Pr}\left(\mathbf{V} \mid H_{k}\right)
$$

This result makes explicit the effect of the correlation. It is easy to see that for uncorrelated sensors we have $\Lambda_{C}(\mathbf{U}) \rightarrow \Lambda_{I}(\mathbf{U})$ as expected.

The effect of sensor correlation can be quantified using Monte Carlo methods as shown in Figure 6. Care is required since the correlation of random normal variables $\mathcal{E}\left[x_{i} x_{j}\right]$ is in general not equal to the desired correlation $\mathcal{E}\left[u_{i} u_{j}\right]$. As noted above for feature correlation, the fusion-derived performance gain decreases rapidly with increasing decision correlation. The correlation levels noted in Table 2 suggest that DL fusion of real sensors may experience only $50 \%$ to $80 \%$ of the performance benefit obtained for independent sensors. 


\section{SUMMARY AND CONCLUDING REMARKS}

Simple theoretical models can be used to predict trends in sensor fusion. These models can be used to qualitatively estimate the performance of fused suites using minimal information including the single-sensor probabilities of detection and false alarm and the hypothesis-conditioned sensor-to-sensor correlations. The probability of error was used to quantify the performance benefit obtained through fusion. This benefit was calculated for both feature-level and decision-level fusion of uncorrelated sensors. We observed that the performance improvement is limited if one sensor in the suite dominates (or is dominated by) the remaining sensors. The peak benefit occurs when all sensors have comparable performance.

Because essentially all demining sensors are clutter limited, and because many sensors respond to similar clutter features, it is reasonable to expect correlation among the outputs of demining sensors. A survey of a few prior experimental works shows that a number of sensors are correlated at levels of $10 \%$ to $50 \%$, with larger correlations observed among features than among decisions. Theoretical calculations of performance for correlated sensors were presented for both feature-level and decision-level fusion. It was observed that correlation greatly reduces the performance improvement attainable through fusion. A comparison of theoretical results and experimentally derived sensor-to-sensor correlations suggests that fusion-related performance gains for real sensors may be significantly smaller than what would be predicted for independent sensors.

\section{ACKNOWLEDGMENTS}

This project was supported by funds from Duke University under an award from the ARO (the OSD MURI program). The findings, opinions and recommendations expressed therein are those of the author and are not necessarily those of Duke University or the ARO.

\section{REFERENCES}

1. K. Fukunaga, Introduction to Statistical Pattern Recognition, Academic Press, Boston, MA, 1990.

2. S. M. Kay, Fundamentals of Statistical Signal Processing: Volume II, Detection Theory, Prentice-Hall, Englewood Cliffs, NJ, 1998.

3. B. A. Baertlein and A. H. Gunatilaka, "Optimizing fusion architectures for limited training data sets," in Detection Technologies for Mines and Minelike Targets V, A. C. Dubey, J. F. Harvey, J. T. Broach, and R. E. Dugan, eds., Proc. SPIE 4038, pp. 804-815, 2000.

4. Z. Chair and P. R. Varshney, "Optimal data fusion in multiple sensor detection systems," IEEE Trans. Aerospace and Electronic Systems AES-22, pp. 98-101, 1986.

5. B. A. Baertlein, W.-J. Liao, and D.-H. Chen, "Fusion of GPR and acoustic LDV sensors," in Detection and Remediation Technologies for Mines and Minelike Targets VI, A. C. Dubey, J. F. Harvey, J. T. Broach, and R. E. Dugan, eds., SPIE 4394, 2001.

6. L. M. Collins, "Statistical signal processing for demining: Experimental validation," tech. rep., Duke University, 1998.

7. P. Gao, L. Collins, J. Moulton, L. Makowsky, R. Weaver, D. Keiswetter, and I. J. Won, "Enhanced detection of landmines using broadband EMI data," in Detection and Remediation Technologies for Mines and Minelike Targets V, A. C. Dubey, J. F. Harvey, J. T. Broach, and R. E. Dugan, eds., SPIE 3710, pp. 2-13, April 5-9 1999.

8. J. M. Sabatier and N. Xiang, "Laser-doppler based acoustic-to-seismic detection of buried mines," in Detection and Remediation Technologies for Mines and Minelike Targets IV, A. C. Dubey, J. F. Harvey, J. T. Broach, and R. Dugan, eds., Proc. SPIE 3710, pp. 215-222, 1999.

9. N. Xiang and J. M. Sabatier, "Land mine detection measurements using acoustic-to-seismic coupling," in Detection and Remediation Technologies for Mines and Minelike Targets V, A. C. Dubey, J. F. Harvey, J. T. Broach, and R. Dugan, eds., Proc. SPIE 4038, pp. 645-655, 2000.

10. V. Aalo and R. Viswanathan, "On distributed detection with correlated sensors: Two examples," IEEE Trans. Aerospace and Electronic Systems AES-25(3), pp. 414-421, 1989.

11. E. Drakopoulos and C. C. Lee, "On distributed detection with correlated sensors: Two examples," IEEE Trans. Aerospace and Electronic Systems AES-27(4), pp. 593-605, 1991. 
12. M. Kam, Q. Zhu, and W. S. Gray, "Optimal data fusion of correlated local decisions in multiple sensor detection systems," IEEE Trans. Aerospace and Electronic Systems AES-28(3), pp. 916-920, 1992.

13. R. S. Blum and S. A. Kassam, "Optimum distributed detection of weak signals in dependent sensors," IEEE Trans. Information Theory IT-38(3), pp. 1066-1079, 1992.

14. R. O. Duda and P. E. Hart, Pattern Classification and Scene Analysis, John Wiley, New York, NY, 1973. 